\title{
Virulence traits associated with Burkholderia cenocepacia ST856 epidemic strain isolated from cystic fibrosis patients
}

Milka Malešević ${ }^{1}$, Zorica Vasiljević ${ }^{2}$, Aleksandar Sovtić ${ }^{3,4}$, Brankica Filipić $^{1,5}$, Katarina Novović ${ }^{1}$, Milan Kojić ${ }^{1}$ and Branko Jovčić ${ }^{1,6^{*}}$ (D)

\begin{abstract}
Background: Burkholderia cenocepacia is considered one of the most problematic cystic fibrosis (CF) pathogens. Colonization prevalence in the Serbian CF population is high and virtually exclusively limited to a single highly transmissible clone of B. cenocepacia ST856 which is positive for both the B. cepacia epidemic strain marker (BCESM) and cable pilin, and is closely related to the epidemic strain CZ1 (ST32).

Methods: Biofilm formation for 182 isolates, and adhesion to components of the host extracellular matrix, proteolytic activity, mucoidy and motility of selected ST856 representatives, as well as B. cenocepacia ST858 and ST859, and B. stabilis ST857, novel STs isolated from Serbian CF patients, were investigated in this study. The presence of the cepl, cepR, fliG, IlpE, wbil, and bcscV genes was analyzed.

Results: Biofilm-formation ability of analyzed strains was poor under standard laboratory conditions, but changed in stress conditions (cold stress) and conditions that mimic CF milieu (increased $\mathrm{CO}_{2}$ ). All strains expressed ability to bind to collagen and fibronectin albeit with different intensity. Representatives of ST856 exhibited gelatinase activity. ST858, ST859 and 9/11 of ST856 genotypes were positive for swimming and twitching motility whereas ST857 was non-motile. Mucoidy was demonstrated in all ST856 genotypes, ST857 was semi-mucoid, and ST858 and ST859 were non-mucoid. Molecular analysis for major virulence factors revealed that ST856 and ST857 carried the six analyzed genes, while ST858 and ST859 were negative for the IIpE gene.

Conclusion: Variations in virulence phenotypes in different genotypes of epidemic B. cenocepacia ST856 clone, in vitro, could be a consequence of diversification driven by pathoadaptation. Diversity of epidemic clone genotypes virulence, could be challenging for accurate diagnosis and treatment, as well as for infection control.
\end{abstract}

Keywords: Burkholderia cenocepacia complex, Cystic fibrosis, Epidemic strain, Virulence

\section{Background}

Bacteria of the Burkholderia cepacia complex (Bcc) have been considered among the most challenging pathogens involved in cystic fibrosis (CF) lung disease [1]. Disparate outcomes of Bcc infections in CF vary from asymptomatic carriage to progressive respiratory deterioration or even rapidly fatal and uncontrollable "cepacia" syndrome [2]. $B$. cenocepacia and B. multivorans are currently the Bcc

\footnotetext{
*Correspondence: bjovcic@bio.bg.ac.rs

'Institute of Molecular Genetics and Genetic Engineering, University of Belgrade, Vojvode Stepe 444a, P.O. Box 23, Belgrade 11010, Serbia

${ }^{6}$ Chair for Biochemistry and Molecular Biology, Faculty of Biology, University of Belgrade, 16, Studentski trg, Belgrade 11000, Serbia

Full list of author information is available at the end of the article
}

species most frequently isolated from clinical samples $[3,4]$. Notably, B. cenocepacia comprises the most virulent and transmissible epidemic clones. Strains belonging to this species had often been reported in association with poor clinical outcome and high mortality among CF patients [1]. During infection of CF patients, Bcc bacteria experience stressful conditions, host immune defense, antimicrobial therapy, nutrient availability and oxygen limitation. Although the process of adaptation to the airways of CF patients is still poorly understood it could be attributed to the undeniable virulence potential of B. cenocepacia and assumption that these bacteria may encode more potent or more 
numerous virulence factors comparing to other Bcc species [5]. Among those are LuxIR homolog CepIR (quorum sensing) that was shown to be widespread in Bcc bacteria and required for full virulence in many Bcc infection models, T3SS, motility (flagellum) and efflux pumps that enhance the process of colonization, adaptation and infection [5]. Bacterial virulence factors are delivered either in the extracellular environment or directly into host cells. Most Gram-negative CF pathogens possess one or more specialized secretion systems to accomplish this task, among which is T3SS. A T3SS mutant of B. cenocepacia was attenuated in virulence in a murine model of infection, which indicates a role for the T3SS in evasion of the host immune system [6]. Also, there are some unique aspects of efflux systems in Burkholderia species that are without parallel. The first gene in the bpeEF-oprC operon, $l l p E$, was shown to be the most prevalent in B. cenocepa$c i a$, and is co-transcribed with the genes encoding the BpeEF-OprC efflux pump components that was shown to be of importance for resistance to antibiotics $[7,8]$. Additionally, the $\mathrm{O}$-antigen portion (coded by the wbil gene) of the LPS molecule is important for resistance of Bcc to serum-mediated killing [9].

However, many of the virulence factors characterized in $B$. cenocepacia appear not to be unique to this species and have homologs in other bacterial pathogens. The detection of two putative epidemic and virulence markers, cable pili [10] and B. cepacia epidemic strain marker (BCESM), had been of particular interest and used to be applied as an infection control measure in limiting the spread of infamous B. cenocepacia ST28 [3, 5, 11]. However, later evidence suggested that neither 'marker' is an accurate indicator of transmissibility or virulence [12]. The $c b l A$ gene is found almost exclusively in strains of the ST28 while BCESM occurs in many different strains from B. cenocepacia [11].

Our previous study evaluated the prevalence and epidemiological characteristics of Bcc strains in the National CF Centre in Belgrade, Serbia [13]. In this study, three novel STs of B. cenocepacia were described (ST856, ST858, and ST859) as well as a novel B. stabilis strain, ST857 [13]. B. cenocepacia ST856 was distinctly dominant and was found in $96 \%$ patients infected with Bcc, and encompassed 11 PFGE pulsotypes [13]. This clone was characterized by PCR positivity for both the B. cepacia epidemic strain marker and cable pilin, and showed close genetic relatedness to the epidemic strain $B$. cenocepacia CZ1 (ST32). B. cenocepacia ST858 and ST859 were isolated from sputum of a single chronically colonized patient. B. stabilis ST857 was also identified in only one patient. An interesting feature of strains BCC267 (ST858) and BCC269 (ST859) was the fact that results of evolutionary distances between concatenated nucleotide sequences of analyzed alleles were not in concordance with
MLST scheme [13]. This analysis grouped ST858 and ST859 within $B$. multivorans, while by MLST they were designated as B. cenocepacia IIIA but as novel STs [12].

Considering the high transmissibility and peculiarity of B. cenocepacia ST856, the aim of this study was to evaluate its virulence potential. We also investigated the virulence potential of other novel STs identified in Serbian CF patients.

\section{Methods}

\section{Bacterial strains}

Bacterial strains used in this study originated from patients $(n=182)$ followed at the National Cystic Fibrosis Centre which is located at the Mother and Child Health Care Institute of Serbia "Dr. Vukan Cupic". All of the strains were characterized previously by our research group [13].

Their genetic relatedness was determined by pulsed-field gel electrophoresis (PFGE) analysis of SpeI macrorestriction profiles. Fourteen different genotypes were identified and they belonged to four different MLST types: $B$. cenocepacia ST856 (11 genotypes), B. cenocepacia ST858 (one genotype), B. cenocepacia ST859 (one genotype) and B. stabilis ST857 (one genotype) [13]. A dendrogram was derived from the Ward linkage of correlation coefficients between PFGE patterns of different genotypes using SPSS cluster analysis software (IBM Corp. Released 2012. IBM SPSS Statistics for Windows, Version 21.0. Armonk, NY: IBM Corp.).

\section{Biofilm production}

Biofilm production was assessed as described previously [14], with modifications. Briefly, one colony of the overnight culture of bacterial strains was adjusted to 0.5 McFarland turbidity standard. Wells of microtiter plates were filled with $180 \mu \mathrm{L}$ of Luria Bertani (LB) medium. $20 \mu \mathrm{L}$ aliquots of previously prepared bacterial suspension were added to each well. Blank wells contained LB medium only. The plates were incubated aerobically for $24 \mathrm{~h}$ at $37^{\circ} \mathrm{C}$. All isolates were tested in triplicate. Pseudomonas aeruginosa $\mathrm{PAO} 1$ and Escherichia coli $\mathrm{DH} 5 \alpha$ were used as positive and negative controls, respectively. After the incubation, wells were emptied and washed three times with $300 \mu \mathrm{l}$ of sterile physiological saline, and then dried at $65{ }^{\circ} \mathrm{C}$. The plates were stained with $0.1 \%$ crystal violet (CV) (HiMedia, India). Thereafter, plates were emptied and dried at $65{ }^{\circ} \mathrm{C}$. The dye bound to the adherent cells was resolubilized with $200 \mu \mathrm{l}$ of ethanol-acetone mixture $(80: 20, v / v)$. The optical density was measured at $595 \mathrm{~nm}$ using Infinite M200 pro (Tecan, Switzerland). Based on the optical densities of bacterial biofilms, all strains were classified as follows:

$\mathrm{OD} \leq \mathrm{ODc}-$ no biofilm producer; $\mathrm{ODc}<\mathrm{OD} \leq 2 \mathrm{xODc}$ - weak biofilm producer; 2xODc $<$ OD $\leq 4 x O D c-$ 
moderate biofilm producer; 4xODc < OD - strong biofilm producer; where ODc is an average value of blank OD with three standard deviations added [14].

\section{Biofilm production under stress conditions}

Biofilm formation was investigated under different stress conditions including dynamic stress $\left(100 \mathrm{rpm}\right.$, at $\left.37^{\circ} \mathrm{C}\right)$, cold stress (growth at $12{ }^{\circ} \mathrm{C}$ ), heat shock (growth at $42{ }^{\circ} \mathrm{C}$ ), increased $\mathrm{CO}_{2}$ levels $\left(5 \% \mathrm{CO}_{2}\right.$, at $37{ }^{\circ} \mathrm{C}$ in HERAcell 150 , Thermo Scientific, USA). Additionally, two different $\mathrm{pH}$ values of LB medium ( $\mathrm{pH} 6.8$ and $\mathrm{pH} 7.45$ ) were used for all tested conditions. Assessment of biofilm production was done as mentioned above. The statistical significance of differences in biofilm production for each stressor was determined in relation to biofilm production of given strain without stress $\left(37^{\circ} \mathrm{C}\right)$ was tested by Student's $t$-test.

\section{Collagen- and fibronectin-binding assays}

The wells of Maxisorb plates (Nunc, Roskilde, Denmark) were coated with type I collagen (from rat tail, BD Bioscience, New Jersey, United States) $(100 \mu \mathrm{g} / \mathrm{ml})$ or human fibronectin (Serva, Heidelberg, Germany) $(100 \mu \mathrm{g} / \mathrm{ml})$ for $16 \mathrm{~h}$ at $4{ }^{\circ} \mathrm{C}$. The collagen-binding ability of the selected strains was tested according to Miljkovic and coauthors [15] while the ability of tested strains to bind to fibronectin was assayed as previously described by Ahmed and coauthors [16]. Average of six absorbance values per each strain for collagen- and fibronectin-binding was compared with those of the non-coated wells. Escherichia coli DH5 $\alpha$ was used as a negative control, while laboratory strain Lactococcus lactis BGKP1 was used as a positive control.

\section{Proteolytic, gelatinase and elastase activity assay}

Proteolytic activity was determined by plating $10 \mu \mathrm{l}$ aliquots of the stationary-phase culture of each strain on medium containing skimmed milk (3\%) and agar (1.5\%). The plates were incubated at $37^{\circ} \mathrm{C}$ for $48 \mathrm{~h}$. Protease producers form transparent halo around the colonies.

Gelatinase activity of representative strains was assessed by previously described method [17]. Transparent halo around colonies was considered as positives for gelatinase production.

The elastase assay was done as described by Jacobson and coauthors [18].

\section{Mucoidy scoring system}

Semiquantitative method for scoring mucoidy among selected strains was performed as previously described [19]. Briefly, mucoidy was assessed on Yeast Mannitol (YEM) agar containing $4 \mathrm{~g} / \mathrm{L}$ mannitol and $0.5 \mathrm{~g} / \mathrm{L}$ yeast extract and 1.5\% agar. Bacteria were subcultured from a frozen stock by using a cotton swab to inoculate one third of a 25-ml YEM plate, streaked to yield individual colonies, and grown at $37{ }^{\circ} \mathrm{C}$ for $48 \mathrm{~h}$. Mucoidy was defined as follows: nonmucoid, semi-mucoid and mucoid.

\section{Motility assays}

Swimming motility was individually assessed for each isolate on plates containing tryptone $(10 \mathrm{~g} / \mathrm{l}), \mathrm{NaCl}(5 \mathrm{~g} / \mathrm{l})$ and $0.3 \%$ (wt/vol) agar as previously described [20]. After $24 \mathrm{~h}$ the diameters of the colonies were measured. Isolates showing growth of less than or equal to $10 \mathrm{~mm}$ in diameter at $24 \mathrm{~h}$ were classified as nonmotile, while those with $>10 \mathrm{~mm}$ were classified as motile.

Medium used for the twitching motility assay consisted of LB broth $(10 \mathrm{~g} / \mathrm{L}$ tryptone; $5 \mathrm{~g} / \mathrm{L}$ yeast extract; $10 \mathrm{~g} / \mathrm{L}$ $\mathrm{NaCl})$ with $1 \%(w / v)$ agar. Twitching plates were briefly dried and strains were stab inoculated with a sharp toothpick to the bottom of the Petri dish from an overnightgrown LB agar $(1.5 \%, w / v)$ plate. After incubation at $37^{\circ} \mathrm{C}$ for $24 \mathrm{~h}$, the zone of motility at the agar/Petri dish interface was measured.

\section{DNA manipulations}

PCR screenings of 6 genetic determinants (cepI, cepR, fliG, llpE, wbiI, bcscV) associated with virulence were performed in accordance with published protocols [21] for representative strains belonging to each genotype of ST856 (eleven) and for ST857, ST858 and ST859. Representative PCR amplicons were selected and sequenced in order to confirm the specificity of the reaction. Sequencing was performed by the Macrogen DNA Sequencing Service (Amsterdam, Netherlands).

\section{Results}

We have analyzed laboratory collection of $182 \mathrm{~B}$. cepacia complex isolates from CF patients in our previous study. Based on PFGE analysis 14 different genotypes were identified. These genotypes belonged to four different MLST types: B. cenocepacia ST856 (11 genotypes), B. cenocepacia ST858 (one genotype), B. cenocepacia ST859 (one genotype) and B. stabilis ST857 (one genotype) [13].

Statistical analysis of PFGE results revealed that genetic differences among genotypes of the epidemic-related $B$. cenocepacia ST856 varied up to approximately 6\% (Fig. 1). This variability pointed to the need of determination of virulence for all defined genotypes of ST856 epidemic clone (11 genotypes).

Biofilm production was analyzed in all 182 isolates from the collection, and the majority of them were weak biofilm producers $(74 \%)$ while only a small proportion produced strong biofilms (3\%) (Table 1). Strong and moderate biofilm producers belonged to various ST856 genotypes, except moderate biofilm-producing strain BCC269 that belonged to ST859. Additionally, we compared biofilm formation capacity between isolates originating from chronically and transiently colonized patients. No statistically significant 


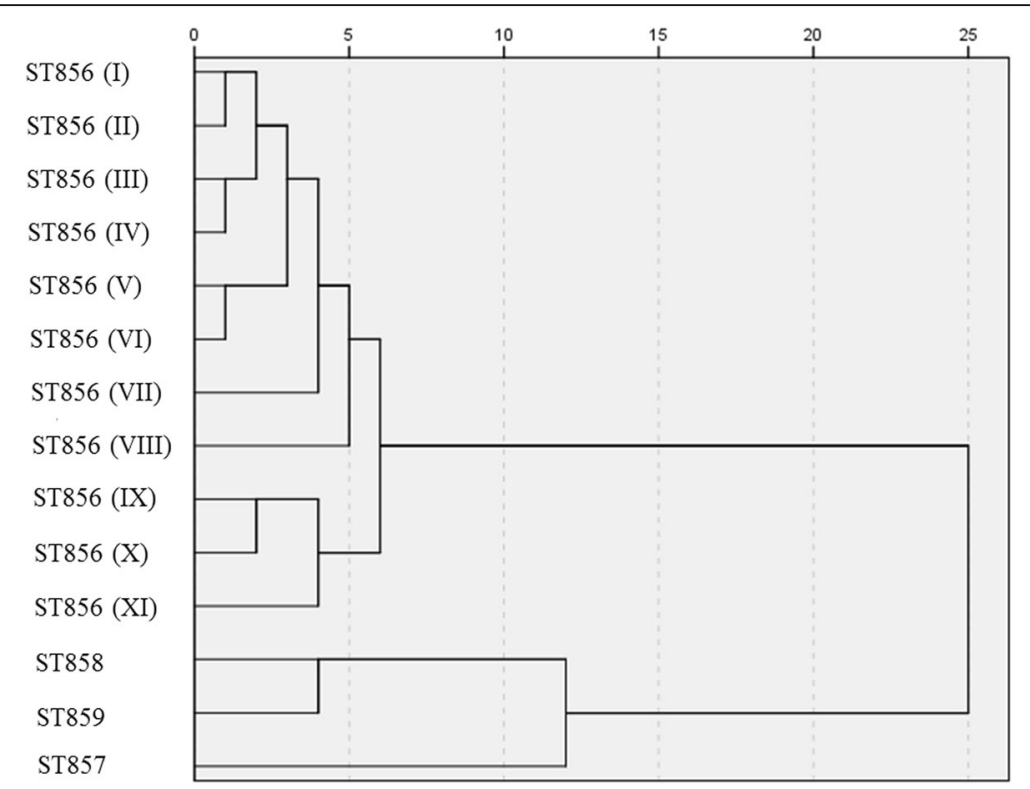

Fig. 1 Dendrogram derived from Spel PFGE patterns showing the relatedness of Burkholderia cepacia complex species isolated from Serbian CF patients. The dendrogram was constructed using SPSS software. Roman numerals indicate different genotypes of B. cenocepacia ST856 epidemic clone

difference was observed between these two groups ( $p=0.787$ and $p>0.5$, respectively) (Table 1$)$. Since these results reflected biofilm production in standard laboratory conditions, we also analyzed biofilm production under stress conditions mimicking those faced by Bcc bacteria in the $\mathrm{CF}$ lung (changes in $\mathrm{pH}$, dynamic stress, elevated $\mathrm{CO}_{2}$ levels) as well as impact of cold and heat shock for the selected representatives of each genotype (14 strains in total). We found that in all representatives of $11 \mathrm{~B}$. cenocepacia ST856 genotypes cold stress $\left(12{ }^{\circ} \mathrm{C}\right)$ and elevated $\mathrm{CO}_{2}$ levels $\left(5 \% \mathrm{CO}_{2}\right)$ enhanced formation of biofilm with statistical significance (0.0001 $<p<0.005$ ) (Fig. 2). Also, the heat stress $\left(42{ }^{\circ} \mathrm{C}\right)$ and the dynamic stress reduced ability of biofilm formation in all representatives of ST856 genotypes with statistical significance $(0.0001<p<0.005)$ (Fig. 2). Changes in $\mathrm{pH}$ values had no influence for all tested isolates (Fig. 2). Biofilm formation of B. cenocepacia ST858 was reinforced under elevated $\mathrm{CO}_{2}$ levels (at both $\mathrm{pH} 6.8$

Table 1 Results of biofilm formation compared to chronic or transient colonisation of the patients (number of isolates $n=182$ )

\begin{tabular}{lllll}
\hline & $\begin{array}{l}\text { Non- } \\
\text { producers }\end{array}$ & \multicolumn{3}{l}{ Biofilm producers } \\
\cline { 3 - 5 } & & Low & Moderate & Strong \\
\hline $\begin{array}{l}\text { Chronic colonisation } \\
(n=161)\end{array}$ & 19 & 118 & 18 & 6 \\
$\begin{array}{l}\text { Transient colonisation } \\
(n=21)\end{array}$ & 2 & 17 & 2 & 0 \\
Total & $21(11.54 \%)$ & $135(74.18 \%)$ & $20(10.99 \%)$ & $6(3.29 \%)$ \\
\hline
\end{tabular}

Percentages are calculated relative to the total number of analysed strains and 7.45; $p=0.0041$ and $p=0.0033$, respectively) (Fig. 2). However, cold stress ( $\mathrm{pH} 6.8, p=0.0079)$, dynamic stress $(\mathrm{pH} 6.8, p=0.0002)$ and heat shock $(\mathrm{pH} \mathrm{6.8,} p=0.0002)$ reduced ability of ST858 to form biofilm. Other experimental conditions had no statistically significant impact on biofilm formation capacity of this strain (Fig. 2). $B$. cenocepacia ST859 more successfully formed biofilm under cold stress (at both pH 6.8 and 7.45; $p<0.0001$ and $p=0.0023$, respectively) and under elevated $\mathrm{CO}_{2}$ levels (at both pH 6.8 and 7.4; $p=0.0008$ and $p=0.0029$, respectively). Additionally, heat shock (at $\mathrm{pH} 6.8 ; p=0.0293$ ) reduced the ability of biofilm formation (Fig. 2). Other experimental conditions had no statistically significant influence on biofilm formation (Fig. 2). Biofilm formation in B. stabillis ST857 was enhanced by increased concentration of $\mathrm{CO}_{2}$ (at both pH 6.8 and 7.45; $p=0.0131$ and $p=0.0002$, respectively) although it was still poor, while other stressors had no statistically significant influence (Fig. 2).

Representatives of all genotypes from this study were tested for the ability to bind to collagen and fibronectin. It was noticed that strains adhered to immobilized collagen (Fig. 3) and fibronectin (Fig. 4) to different extents. Interestingly, differences in adherence to immobilized collagen and fibronectin were apparent between strains of different genotypes of $B$. cenocepacia ST856. In most strains, adherence to fibronectin and collagen correlated (Fig. 5).

Proteolytic activity testing, performed with skimmed milk, revealed that eight out of 11 of epidemic ST856 genotypes exhibited proteolytic activity (genotypes I, II, 

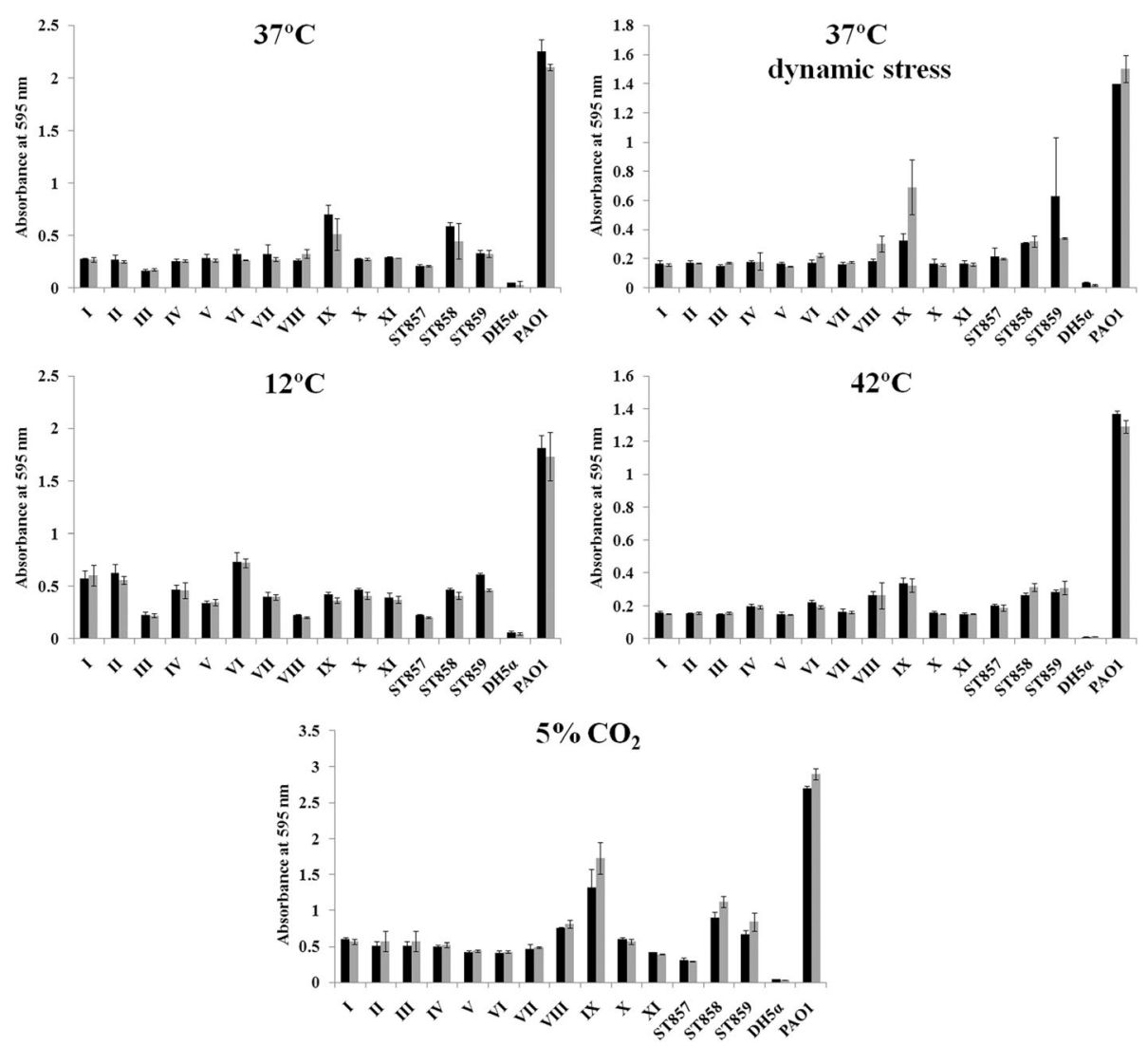

Fig. 2 Biofilm formation of representative strains of different genotypes of Burkholderia cepacia complex isolated from Serbian CF patients under conditions that mimic milieu of CF lung and stress conditions. Dark grey histograms represent results for pH 6.8, light grey pH 7.45. Roman numerals indicate different genotypes of B. cenocepacia ST856 epidemic clone. Applied stress conditions are indicated in each graph. DH5anegative control, PAO1 - positive control

and XI did not form a transparent halo). B. stabilis ST857 was proteolytically inactive while $B$. cenocepacia ST858 and ST859 formed transparent halo, demonstrating proteolysis of milk proteins. None of the strains were able to hydrolyze elastine. Gelatinase activity was detected in all genotypes of ST856, while representatives of ST857, ST858, and ST859 were inactive in this assay (Fig. 5).

Mucoidy was tested by semiquantitative method in representatives of 14 genotypes. All genotypes of the ST856 clone were mucoid except genotype $\mathrm{X}$ that was

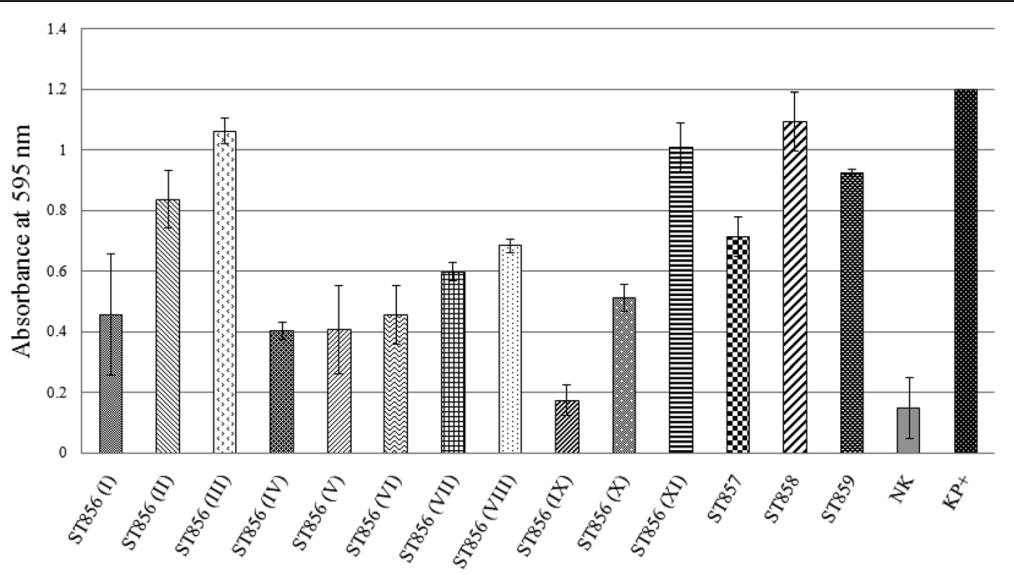

Fig. 3 Collagen binding ability of B. cenocepacia ST856, B. cenocepacia ST858, B. cenocepacia ST859, and B. stabilis ST857. Roman numerals indicate different genotypes of B. cenocepacia ST856 epidemic clone. NK - E. coli DH5a, KP+ - L. lactis BGKP1 


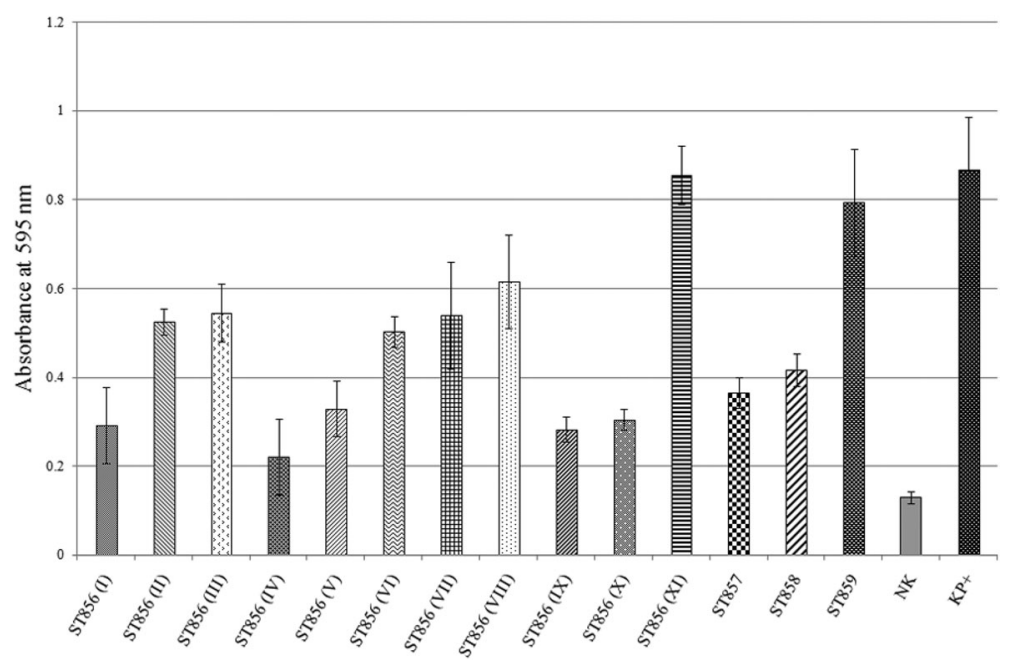

Fig. 4 Fibronectin binding ability of B. cenocepacia ST856, B. cenocepacia ST858, B. cenocepacia ST859, and B. stabilis ST857. Roman numerals indicate different genotypes of B. cenocepacia ST856 epidemic clone. NK - E. coli DH5a, KP+ - L. lactis BGKP1

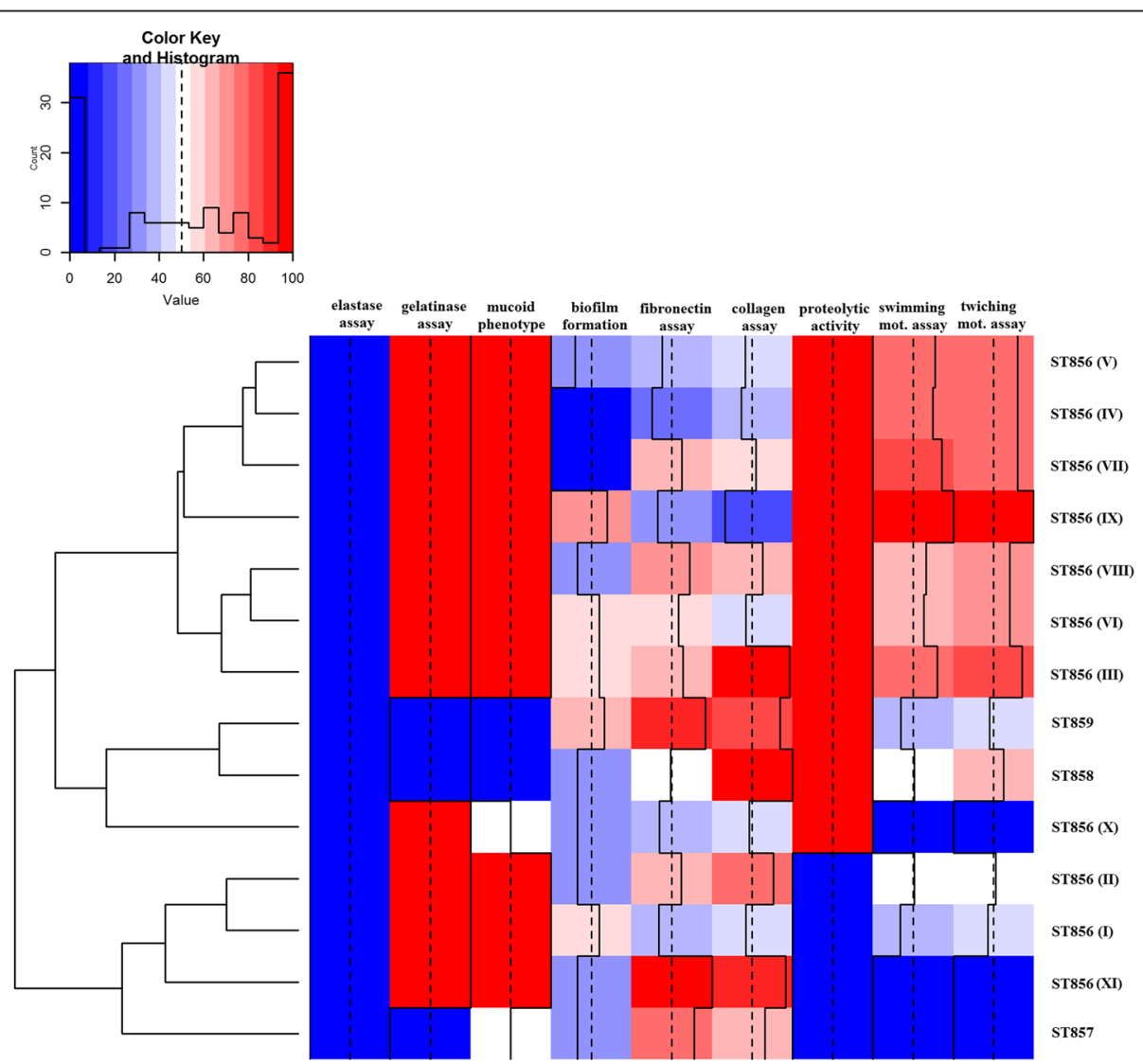

Fig. 5 Heat-map describing presence of different virulence phenotypes of B. cenocepacia ST856, B. cenocepacia ST858, B. cenocepacia ST859, and B. stabilis ST857. Roman numerals indicate different genotypes of B. cenocepacia ST856 epidemic clone 
semi-mucoid. B. stabilis ST857 was also semi-mucoid, while B. cenocepacia ST858 and ST859 were nonmucoid (Fig. 5).

Given the well-described correlation between the loss of swimming motility and establishment of chronic CF lung infections, we assessed swimming motility of bacteria of each genotype in order to understand the significance of swimming motility during Bcc infections. Nine out of 11 ST856 different genotype representatives were motile (zone of migration ranging from 12 to $35 \mathrm{~mm}$ ), while genotypes $\mathrm{X}$ and XI were non-motile. B. stabilis ST857 was nonmotile, whereas B. cenocepacia ST858 (18 mm) and ST859 (12 mm) could be considered as motile. Similar results were obtained for ST856 genotype representatives in twitching motility assay where nine out of 11 were positive (zone ranging from 17 to $40 \mathrm{~mm}$ ). Genotypes $\mathrm{X}$ and XI were non-motile in twitching motility assay. B. stabilis ST857 was non motile, while B. cenocepacia ST858 $(25 \mathrm{~mm})$ and ST859 (18 $\mathrm{mm})$ were motile.

Since quorum sensing, flagellum, some efflux-pumps and type-III secretion system were previously correlated with virulence, PCR screening for six genetic determinants (cepI, cepR, fliG, llpE, wbiI, and bcscV) was performed. Results revealed that B. cenocepacia ST856 strains and $B$. stabilis ST857 were positive for all analyzed genes. B. cenocepacia ST858 and ST859 were negative only for the llpE gene that is a part of the CeoAB-OpcM efflux pump operon.

Heat map demonstrating distribution of virulence characteristics analyzed in this study was constructed in order to summarize virulence potential of different genotypes of B. cenocepacia ST856, as well as B. cenocepacia ST858, ST859 and B. stabilis ST857 (Fig. 5). To compute a dendrogram, we used clustering algorithm that groups related rows together by similarity. Results were approximated on the relative scale ranging from 0 (blue) as the lowest value, progressing to white, then to 100 (red) as the highest value. Analyzed strains were separated into three clusters that reflect virulence potential. The heat-map revealed that gelatinase production, proteolytic activity, mucoidy, motility and adhesion to fibronectin and collagen were the most prominent characteristics of ST856 epidemic clone genotypes III, IV, V, VI, VII, VIII and IX strains were clustered together. ST857 was clustered with genotypes I, II and XI of ST856, since were phenotypically negative for most of the analyzed properties, with exception of adhesion to fibronectin and collagen and variable mucoidy appearance. ST859, ST858 and genotype X of ST856 were clustered together and on the basis of the positivity in applied phenotypic tests were grouped between previously mentioned clusters.

\section{Discussion}

Considering the fact that in Serbian CF patients Bcc colonization prevalence reaches over $20 \%$ and that a single prevailing $B$. cenocepacia ST856 epidemic clone was identified in $96 \%$ of the patients infected with Bcc [13], the determination of its overall virulence potential has imposed as a potentially useful aid in understanding its successful dissemination and high prevalence. Divergence of 11 ST856 genotypes, which differed up to 6\% according to PFGE analysis, raised the question of whether they possess common/distinct virulence potential. Among other virulence factors, ability to form biofilms is particularly intriguing since it contributes significantly to Bcc resistance to antimicrobials and antiseptics, resulting in treatment failure and persistence of infection [22]. Assessment of biofilm production in standard laboratory conditions revealed the low-level potential of ST856 genotypes. However, this observation may not reflect the real biofilmforming potential of these bacteria, as in vivo bacterial biofilms are formed in a complex interaction with surrounding tissues and immune system of the host [23]. Additionally, novel findings indicate that Bcc bacteria are predominantely in the form of single cells or small clusters within phagocytes and mucus in late-stage CF lung, thus not forming biofilms [24]. These data suggest that Bcc bacteria have adapted as single-cell organisms, but not biofilms, to environments in the CF lung [24]. Thus formation of biofilm seems not to be crucial for pathogenicity of Bcc in CF lungs, and the low-level biofilm-forming potential of ST856 genotypes doesn't mean that they are not well adapted for infection of CF-lungs. Previous reports concluded that B. cenocepacia strains, especially those belonging to genomovar III-A which encompasses ST856, ST858 and ST859 [12], could generally be considered avid biofilm producers [25]. Thus, it was of interest to determine whether the conditions that mimic the milieu of CF airways or additional stress conditions influence the potential for biofilm formation of these strains. Cold stress and increased concentration of $\mathrm{CO}_{2}$ enhanced biofilm formation by all ST856 genotypes indicating the possible significance of such experiments.

It has been previously postulated that the ability of BcC to adapt and survive in acidic environment might be necessary for successful colonization of the CF lung due to fact that the $\mathrm{pH}$ of exhaled breath condensate is lower in patients with stable CF than in healthy controls, and is further reduced in CF patients with an infective exacerbation [26-28]. We demonstrated that, in the applied range, the $\mathrm{pH}$ value of the medium did not affect biofilm formation in analyzed ST856 genotypes. However, statistically significant influence of $\mathrm{pH}$ value on biofilm formation was detected in B. cenocepacia ST858 and ST859. These observations indicate that the response to decreased $\mathrm{pH}$ value is probably strain dependent. B. stabilis ST857 was 
shown to be a weak-biofilm producer, and this characteristic was not affected under different stress conditions except for increased concentration of $\mathrm{CO}_{2}$. This result is in accordance with the previously published results regarding this species [29]. Although all of the ST856 genotypes contained the cepI-cepR quorum sensing system, they exerted differences in the level of biofilm formation. This is not entirely surprising as correlation between the presence of quorum sensing and biofilm formation has been questioned previously. The strains lacking this correlation were described, such as B. cenocepacia ST28 with inactivated cepI autoinducer which was still able to form biofilm [29]. Molecular detection of virulence determinants revealed that $B$. cenocepacia ST858 and ST859 lacked the llpE gene encoding salicylate-regulated antibiotic efflux operon. This operon has been identified in B. cenocepacia, and the $l l p E$ gene is without parallel in previously reported efflux operons. The $l l p E$ gene was shown to be the most prevalent in B. cenocepacia, with a high degree of sequence conservation $[7,8]$. The lack of this B. cenocepacia marker in genomes of ST858 and ST859 is particularly interesting in view of the unusual/unique phylogenetic status of these strains, as the results of evolutionary distances between concatenated nucleotide sequences of seven alleles that grouped the two STs close to B. multivorans were not in concordance with MLST scheme which identified them as B. cenocepacia [13].

Another virulence trait which may contribute to the severity of Bcc infections is the ability to secrete extracellular proteases, as reported for a number of Bcc species [30]. Proteolytic activity of the strains analyzed in this study varied among different genotypes of ST856, and $3 / 11$ were proteolytically inactive. However, these results should be interpreted cautiously, as Bcc species could be phenotypically negative for proteolysis when skim milk agar is used as a substrate, even when the zmpA gene encoding the most common extracellular protease of these bacteria is present in the genome [30]. Mucoidy testing revealed that all genotypes of ST856 were mucoid, except genotype $\mathrm{X}$ that was semi-mucoid. This feature may contribute to the pathogenicity of the ST856 epidemic clone since the intensity of mucoidy was previously shown to be correlated with the rate of decline in pulmonary function as well as with increased virulence [31-33]. Also, mucoid isolates from CF patients had reduced interaction with macrophages and neutrophils enabling evasion of the host response [34]. However, it is worth noting that some exceptions exist, since all isolates of the virulent ST28 described to date are non-mucoid [19]. Additionally, it is known that Bcc strains change from a mucoid to a non-mucoid phenotype during chronic colonisation and patients which were infected exclusively with non-mucoid Bcc had a more rapid decline in lung function than those infected with mucoid bacteria [34].

\section{Conclusions}

Our data suggest that detecting variations in virulence potential of different Serbian epidemic clone B. cenocepacia ST856 genotypes might be useful for risk assessment. Although all identified ST856 genotypes carry $c b l A$ and BCESM markers [13], phenotypic differences were observed regarding other virulence factors. This is not completely unexpected since B. cenocepacia is a member of a highly adaptable genus of bacteria that can rapidly evolve under in vitro stress conditions or during infections [35]. This adaptability may be the key in explaining differences observed among B. cenocepacia ST856 genotypes. Similarly, variability of virulence among the same genetic line for virulence was previously documented for the cable pili [35]. Diversity of epidemic clone genotypes virulence, could be challenging for accurate diagnosis and treatment, as well as for infection control. Thus, different genotypes of epidemic strains should be taken into account during decision making on cohort segregation of CF patients with Bcc infection.

\section{Abbreviations}

Bcc: Burkholderia cepacia complex; CF: Cystic fibrosiss; BCESM: Burkholderia cepacia epidemic strain marker; MLST: Multilocus sequence typing;

PFGE: Pulsed-field gel electrophoresis; LB: Luria Bertani; YEM: Yeast Mannitol

\section{Acknowledgement}

Not applicable.

\section{Funding}

This work was supported by grant No. 173019 from the Ministry of Education, Science and Technological Development of the Republic of Serbia.

\section{Availability of data and materials}

The data and the materials are freely available to any scientist wishing to use them.

\section{Authors' contributions}

$M M, Z V, A S, B F, K N$ were involved in acquisition of laboratory and medical data, analysis of data and final approval of manuscript; MK was involved in data analysis, drafting of article, critical revison of article and final approval of manuscript; BJ designed the study, was involved in analysis of data, drafting of article and final approval of manuscript.

\section{Competing interests \\ The authors declare that they have no competing interests.}

\section{Consent for publication \\ Not applicable.}

Ethics approval and consent to participate Not applicable.

\section{Publisher's note}

Springer Nature remains neutral with regard to jurisdictional claims in published maps and institutional affiliations. 


\section{Author details}

Institute of Molecular Genetics and Genetic Engineering, University of Belgrade, Vojvode Stepe 444a, P.O. Box 23, Belgrade 11010, Serbia. ${ }^{2}$ Department of Clinical Microbiology, Mother and Child Health Care Institute of Serbia "Dr. Vukan Cupic", Radoja Dakica 8, Belgrade 11070, Serbia. ${ }^{3}$ Department of Pulmonology, Mother and Child Health Care Institute of Serbia "Dr. Vukan Cupic", Radoja Dakica 8, Belgrade 11070, Serbia. "School of Medicine, University of Belgrade, Dr Subotica 8, Belgrade 11000, Serbia. ${ }^{5}$ Faculty of Pharmacy, University of Belgrade, Vojvode Stepe 450, Belgrade 11221, Serbia. ${ }^{6}$ Chair for Biochemistry and Molecular Biology, Faculty of Biology, University of Belgrade, 16, Studentski trg, Belgrade 11000, Serbia.

Received: 1 February 2017 Accepted: 30 May 2017 Published online: 06 June 2017

\section{References}

1. Folescu TW, da Costa CH, Cohen RWF, da Conceição Neto OC, Albano RM, Marques EA. Burkholderia cepacia complex: clinical course in cystic fibrosis patients. BMC Pul Med. 2015;15:158. doi:10.1186/s12890-015-0148-2.

2. Mahenthiralingam E, Baldwin A, Vandamme P. Burkholderia cepacia complex infection in patients with cystic fibrosis. J Med Microbiol. 2002;51:1-6.

3. Drevinek P, Mahenthiralingam E. Burkholderia cenocepacia in cystic fibrosis: epidemiology and molecular mechanisms of virulence. Clin Microbiol Infect. 2010;16:821-30

4. Coenye T, Vandamme P. Diversity and significance of Burkholderia species occupying diverse ecological niches. Environ Microbiol. 2003:5:719-29.

5. Denniss JJ. Burkholderia cenocepacia virulence microevolution in the CF lung: variations on a theme. Virulence. 2016;27:1-3. doi:10.1080/21505594.2016.

6. Tomich M, Griffith A, Herfst CA, Burns JL, Mohr CD. Attenuated virulence of a Burkholderia cepacia type III secretion mutant in a murine model of infection. Infect Immun. 2003;71:1405-15.

7. Nair BN, Joachimiak LA, Chattopadhyay S, Montano I, Burns J. Conservation of a novel protein associated with an antibiotic efflux operon in Burkholderia cenocepacia. FEMS Microbiol Lett. 2005;245:337-44.

8. Podnecky NL, Rhodes KA, Schweizer HP. Eflux pump-mediated drug resistance in Burkholderia. Front Microbiol. 2015;6:305. doi:10.3389/fmicb. 2015.00305.

9. Ortega X, Hunt TA, Loutet S, Vinion-Dubiel AD, Datta A, Choudhury B, et al. Reconstitution of O-specific lipopolysaccharide expression in Burkholderia cenocepacia strain J2315, which is associated with transmissible infections in patients with cystic fibrosis. J Bacteriol. 2005;187:1324-33.

10. Goldberg JB, Ganesan S, Comstock AT, Zhao Y, Sajjan US. Cable pili and the associated $22 \mathrm{kDa}$ adhesin contribute to Burkholderia cenocepacia persistence in vivo. PLoS One. 2011;6(7):e22435.

11. Speert DP, Henry D, Vandamme P, Corey M, Mahenthiralingam E. Epidemiology of Burkholderia cepacia complex in patients with cystic fibrosis, Canada. Emerg Infect Dis. 2002;8:181-7.

12. Govan JR, Brown AR, Jones AM. Evolving epidemiology of Pseudomonas aeruginosa and the Burkholderia cepacia complex in cystic fibrosis lung infection. Future Microbiol. 2007;2(2):153-64

13. Vasiljevic Z, Novovic K, Kojic M, Sovtic A, Minic P, Djukic S, et al. Burkholderia cepacia complex in Serbian patients with cystic fibrosis: prevalence and molecular epidemiology. Eur J Clin Microbiol Infect Dis. 2016; doi:10.1007/ s10096-016-2662-4.

14. Stepanović S, Vuković D, Dakić I, Savić B, Švabić-Vlahović M. A modified microtiter-plate test for quantification of staphylococcal biofilm formation. J Microbiol Methods. 2000;40(2):175-9.

15. Miljkovic M, Strahinic I, Tolinacki M, Zivkovic M, Kojic S, Golic N, et al. AggLb is the largest cell-aggregation factor from Lactobacillus paracasei subsp. paracasei BGNJ1-64, functions in collagen adhesion, and pathogen exclusion in vitro. PLoS One. 2015;10:e0126387. doi:10.1371/journal.pone.0126387.

16. Ahmed S, Meghji S, Williams RJ, Henderson B, Brock JH, Nair SP. Staphylococcus aureus fibronectin binding proteins are essential for internalization by osteoblasts but do not account for differences in intracellular levels of bacteria. Infect Immun. 2001;69:2872-7.

17. Lopes MF, Simões AP, Tenreiro R, Marques JJ, Crespo MT. Activity and expression of a virulence factor, gelatinase, in dairy enterococci. Int J Food Microbiol. 2006;112(3):208-14.

18. Jakobsen $\mathrm{TH}$, Hansen MA, Jensen $\mathrm{P} \varnothing$, Hansen L, Riber L, Cockburn A, et al. Complete genome sequence of the cystic fibrosis pathogen Achromobacter xylosoxidans NH44784-1996 complies with important pathogenic phenotypes. PLoS One. 2013;8(7):e68484. doi:10.1371/journal.pone.0068484.

19. Zlosnik J, Hird T, Fraenkel M, Moreira L, Henry D, Speert D. Differential mucoid exopolysaccharide production by members of the Burkholderia cepacia complex. J Clin Microbiol. 2008;46(4):1470-3.

20. Trancassini $M$, lebba $V$, Citerà $N$, Tuccio $V$, Magni A, Varesi $P$, et al. Outbreak of Achromobacter xylosoxidans in an Italian cystic fibrosis center: genome variability, biofilm production, antibiotic resistance, and motility in isolated strains. Front Microbiol. 2014:5:138. doi:10.3389/fmicb.2014.00138.

21. Graindorge A, Menard A, Neto M, Bouvet C, Miollan R, Gaillard S, et al. Epidemiology and molecular characterization of a clone of Burkholderia cenocepacia responsible for nosocomial pulmonary tract infections in a french intensive care unit. Diagn Microbiol Infect Dis. 2008;66:29-40.

22. Sousa S, Ramos CG, Leitão JH. Burkholderia cepacia complex: emerging multihost pathogens equipped with a wide range of virulence factors and determinants. Int J Microbiol. 2011; doi:10.1155/2011/607575.

23. Bjarnsholt T. The role of bacterial biofilms in chronic infections. APMIS 2013: Suppl (136):1-51. http://www.ncbi.nlm.nih.gov/pubmed/23635385.

24. Schwab U, Abdullah LH, Perlmutt OS, Albert D, Davis CW, Arnold RR, et al. Localization of Burkholderia cepacia complex bacteria in cystic fibrosis lungs and interactions with Pseudomonas aeruginosa in hypoxic mucus. Infect Immun. 2014:82(11):4729-45.

25. Conway BD, Venu V, Speert DP. Biofilm formation and acyl homoserine lactone production in the Burkholderia cepacia complex. J Bacteriol. 2002; 184(20):5678-85

26. Tate S, MacGregor G, Davis M, Innes JA, Greening AP. Airways in cystic fibrosis are acidified: detection by exhaled breath condensate. Thorax. 2002;57:926-9.

27. Konstantinidi EM, Lappas AS, Tzortzi AS, Behrakis PK. Exhaled breath condensate: technical and diagnostic aspects. Sci World J. 2015;435160:25 http://dx.doi.org/10.1155/2015/435160

28. Pezzulo AA, Tang XX, Hoegger MJ, Abou Alaiwa MH, Ramachandran S, Moninger TO, Karp PH, Wohlford-Lenane CL, Haagsman HP, van Eijk M, Bánfi B, Horswill AR, Stoltz DA, McCray PB Jr, Welsh MJ, Zabner J. Reduced airway surface $\mathrm{pH}$ impairs bacterial killing in the porcine cystic fibrosis lung. Nature 2012;487(7405):109-113. doi: 10.1038/nature11130.

29. Schwab U, Leigh M, Ribeiro C, Yankaskas J, Burns K, Gilligan P, et al. Patterns of epithelial cell invasion by different species of the Burkholderia cepacia complex in well-differentiated human airway epithelia. Infect Immun. 2002; 70(8):4547-55.

30. Gingues S, Kooi C, Visser MB, Subsin B, Sokol PA. Distribution and expression of the ZmpA metalloprotease in the Burkholderia cepacia complex. J Bacteriol. 2005;187(24):8247-55.

31. Conway BA Chu KK, Bylund J, Altman E, Speert DP. Production of exopolysaccharide by Burkholderia cenocepacia results in altered cell surface interactions and altered bacterial clearance in mice. J Infect Dis. 2004;190:957-66.

32. Silva IN, Ferreira AS, Becker JD, Zlosnik JE, Speert DP, He J, et al. Mucoid morphotype variation of Burkholderia multivorans during chronic cystic fibrosis lung infection is correlated with changes in metabolism, motility, biofilm formation and virulence. Microbiology. 2011;157(Pt 11):3124-37. doi: 10.1099/mic.0.050989-0.

33. Cullen L, McClean S. Bacterial adaptation during chronic respiratory infections. Pathogens. 2015;4(1):66-89. doi:10.3390/pathogens4010066.

34. Zlosnik JE, Costa PC, Brant R, Hird TJ, Mori PY, Wilcox P, et al. Exopolysaccharide production in BCC and links to clinical outcome. Seattle: International Burkholderia cepacia Working Group; 2010. Abstract 7B

35. Drevinek $P$, Baldwin A, Lindenburg L, Joshi LT, Marchbank A, Vosahlikova S, et al. Oxidative stress of Burkholderia cenocepacia induces insertion sequence-mediated genomic rearrangements that interfere with macrorestriction-based genotyping. J Clin Microbiol. 2010;48:34-40. 